\title{
Reciprocal regulatory mechanism between miR-214-3p and FGFR1 in FGFR1-amplified lung cancer
}

Ying Yang ${ }^{1,2}$, Ziming $\mathrm{Li}^{1}$, Hong Yuan', Wenxiang Ji ${ }^{1}$, Kaixuan Wang ${ }^{1}$, Tingting Lu' ${ }^{1}$, Yongfeng Yu', Qingyu Zeng ${ }^{2}$, Fan $\mathrm{Li}^{2}$, Weiliang Xia $\mathbb{C}^{2}$ and Shun $\mathrm{Lu}^{1}$

\begin{abstract}
MicroRNA (miRNA) and fibroblast growth factor receptor 1 (FGFR1) dysregulation are considered to play an important role in tumor proliferation, invasion, and metastasis. However, the regulatory mechanism between miRNAs and FGFR1 in lung cancer remains unclear and extremely critical. miR-214-3p was sharply decreased and showed a significantly negative correlation with FGFR1 in lung cancer patients $(n=30)$. Luciferase reporter assay confirmed that miR-214-3p could downregulate FGFR1 by directly targeting 3'-untranslated region (UTR). miR-214-3p inhibited the processes of epithelial-mesenchymal transition and Wnt/MAPK/AKT (Wnt/mitogen-activated protein kinase/AKT) signaling pathway by targeting FGFR1. Moreover, miR-214-3p not only established a negative feedback regulation loop with FGFR1 through ERK (extracellular signal-regulated kinase) but also developed a synergism with FGFR1 inhibitor AZD4547. In conclusion, our study demonstrated the regulatory mechanism between miR-214-3p and FGFR1 in lung cancer. miR-214-3p acts as a vital target in FGFR1-amplified lung cancer by forming a miR-214-3p-FGFR1-Wnt/MAPK AKT signaling pathway network. Co-targeting miR-214-3p and FGFR1 could provide greater benefits to patients with FGFR1-amplified lung cancer.
\end{abstract}

\section{Introduction}

Worldwide, lung cancer with a 5-year survival rate of $18 \%$ leads the cancer incidence and mortality, and accounts for almost $20 \%$ cancer deaths ${ }^{1,2}$. Lung cancer is mainly composed of small-cell lung cancer (SCLC 10-15\%) and non-small-cell lung cancer (NSCLC, $80-85 \%)$. NSCLC is subdivided into adenocarcinoma, squamous cell carcinoma (SQCC), large cell carcinoma, and so on $^{3,4}$. Early studies have already demonstrated that local progression and distant metastasis, possibly associated with epithelial-mesenchymal transition (EMT), are the most common obstacles in the treatment of lung cancer ${ }^{5}$. Targeted drugs have been developed in

\footnotetext{
Correspondence: Weiliang Xia (wlxia@sjtu.edu.cn) or Shun Lu (shunlu@sjtu.edu.cn) ${ }^{1}$ Shanghai Lung Cancer Center, Shanghai Chest Hospital, Shanghai Jiao Tong University, West Huaihai Road 241, 20030 Shanghai, China

${ }^{2}$ School of Biomedical Engineering and Med-X Research Institute, Shanghai Jiao Tong University, Huashan Road 1954, 200030 Shanghai, China
}

lung adenocarcinoma (LADC) to inhibit tumor proliferation and invasion. The discovery of molecularly targeted therapies for patients with EGFR mutation and ALK or ROS1 rearrangements has been a breakthrough in the treatment history of LADC 6 . However, only a few genomic alterations of lung squamous cell carcinoma (LSQCC) and SCLC have been discovered, and the corresponding molecularly targeted drugs have not been used for clinical treatment ${ }^{7}$. Fibroblast growth factor receptor (FGFR) dysfunction is fairly common in a variety of cancers ${ }^{8}$. Meanwhile, FGFR1 amplification is the most common type, with $20 \%$ in LSQCC, $5-7 \%$ in SCLC, and 1-3\% in LADC ${ }^{8-11}$. AZD4547 and BGJ398 are selective inhibitors of FGFR1, 2, and 3 in phase Ib clinical trials ${ }^{12-16}$, whereas LY2874455 is a pan-FGFR inhibitor in phase I clinical trials ${ }^{17,18}$. FGFR1-amplified lung cancer cell lines have shown promising preclinical sensitivity to kinase inhibition ${ }^{19-21}$. However, the effect 
of these FGFR1 inhibitors are modest. It is known that FGFR1 can facilitate tumor development by promoting EMT in various cancers, including lung cancer, gastric cancer, prostate cancer, and breast cancer ${ }^{16,22-24}$. EMT is also considered to be one of the key factors for drug resistance ${ }^{25}$. Therefore, as a promising target in LSQCC and SLCL, there is an urgent need to further explore the development and progression of FGFR1 in FGFR1amplified lung cancer.

MicroRNAs (miRNAs) are a class of endogenous and small non-coding RNAs (20-24 nucleotides) that regulate a large variety of cellular processes, including differentiation, apoptosis, and proliferation, as well as EMT ${ }^{26-29}$. Through the binding to the $3^{\prime}$-untranslated region $\left(3^{\prime}\right.$-UTR) of target genes, miRNAs could function as either tumor suppressors or promoters ${ }^{30}$. miRNAs are dysregulated in many types of tumors ${ }^{31}$. To date, studies have unveiled a large amount of miRNA signatures in lung cancer, yet miRNA dysregulation in FGFR1-amplified lung cancer has remained unclear. Therefore, it is necessary to explore the regulatory mechanism between miRNAs and FGFR1 in FGFR1-amplified lung cancer, which may help explain the modest suppression of lung cancer by FGFR1 inhibitors, and, more importantly, develop novel and better therapies.

\section{Results}

\section{Reduced miR-214-3p expression confers poor survival}

To determine the correlation between miRNA and the progression of lung cancer, we examined 10 metastasisassociated miRNAs in 30 patients with LSQCC (Fig. S1). Quantitative real-time PCR (qRT-PCR) demonstrated a lower expression of miR-214-3p in the tumor tissues of lung cancer $(n=30)$ than in non-tumor adjacent tissues (NATs) (Fig. 1a), while immunohistochemistry (IHC) showed a higher FGFR1 level in the tumor tissues of lung cancer than in the NATs (Fig. 1d). A Kaplan-Meier analysis verified longer overall survival (OS) in LSQCC patents with high miR-214-3p in The Cancer Genome Atlas (TCGA) database (Fig. 1b). The miRNA profiles of SCLC in GSE27435 also demonstrated that longer OS in SCLC patients with high miR-214-3p (Fig. 1c). Negative correlation between miR-214-3p and FGFR1 was confirmed by qRT-PCR (Fig. 1e). The above results revealed that both miR-214-3p and FGFR1 are involved in lung cancer progression.

\section{miR-214-3p suppressed the proliferation, migration, and invasion of FGFR1-amplified lung cancer cells}

To explore the role of miR-214-3p in FGFR1-amplified lung cancer cells, H1581, DMS114, and HCC95 cells with high expression of FGFR1 were used and were authenticated by short tandem repeat (STR) profiling (Table $\mathrm{S} 1)^{19-21}$. Both cells were transfected with miR-214-3p mimics or inhibitors. miR-214-3p inhibited proliferation in all of the cell lines, as determined by the CCK8 assay and colony formation (Fig. 2a, b). miR-214-3p inhibited migration and invasion as determined by scratch assay and transwell assay (Fig. 2c, d). The altered morphological characteristics of the cells caused by the inhibition of EMT process by miR-214-3p were presented (Fig. 2e). Moreover, miR-214-3p overexpression resulted in the downregulation of mesenchymal markers including vimentin (VIM) and Snail, as well as the upregulation of epithelial markers such as E-cadherin (E-cad) and ZO-1 in both $\mathrm{H} 1581$ and DMS114 cells (Fig. 2f).

Conversely, the downregulation of miR-214-3p promoted proliferation, migration, and invasion in both cell lines (Fig. S2), inhibited the levels of epithelial markers, and induced the expression of mesenchymal markers (Fig. S2).

These results indicated that miR-214-3p blocked the EMT process in FGFR1-amplified lung cancer cell lines as a tumor suppressor.

\section{FGFR1 is a direct target of miR-214-3p}

miRWalk 2.0 and TargetScan were used to predict the potential targets and potential binding sites of miR-214$3 p$, and hundreds of genes were screened out. Combined with a literature search $\left(\mathrm{PubMed}^{\odot}{ }^{\odot}, \mathrm{NCBI}\right.$, Bethesda, MD, USA), we found the 3 '-UTR of FGFR1 with potential miR214-3p binding sites (Fig. 3a). Meanwhile, FGFR1 had a sharp downregulation after miR-214-3p overexpression for $48 \mathrm{~h}$, which was detected by western and qRT-PCR (Fig. 3b, c). To test whether the miR-214-3p directly regulates FGFR1, mutant (FGFR1 3'-UTR-MUT) 3'-UTR, and wild type (FGFR1 $3^{\prime}$-UTR-WT), the regions of FGFR1 gene were separately cloned into the pGL3 vector downstream of the luciferase-coding region. The luciferase reporter assay indicated that miR-214-3p reduced the luciferase activity of the WT 3 '-UTR reporter in all of the cell lines, but had no effect on the MUT 3'-UTR reporter (Fig. 3d-f), which suggested that FGFR1 expression was directly regulated by miR-214-3p.

\section{miR-214-3p inhibits proliferation, migration, and invasion of NSCLC cells by targeting FGFR1}

To determine whether FGFR1 acts a pivotal role in miR-214-3p-induced alterations in cell proliferation and metastasis, H1581, DMS114, and HCC95 cells transfected with FGFR1 overexpression plasmid were used. The inhibitory effects of miR-214-3p on proliferation (Fig. 4a, b) and metastasis (Fig. 4c) induced by miR-214$3 p$ were abrogated by FGFR1 overexpression in the cells. The downregulation of mesenchymal markers, including VIM and Snail, and the upregulation of epithelial marker, E-cad induced by miR-214-3p, were also rescued by FGFR1 restoration (Fig. 4d). 

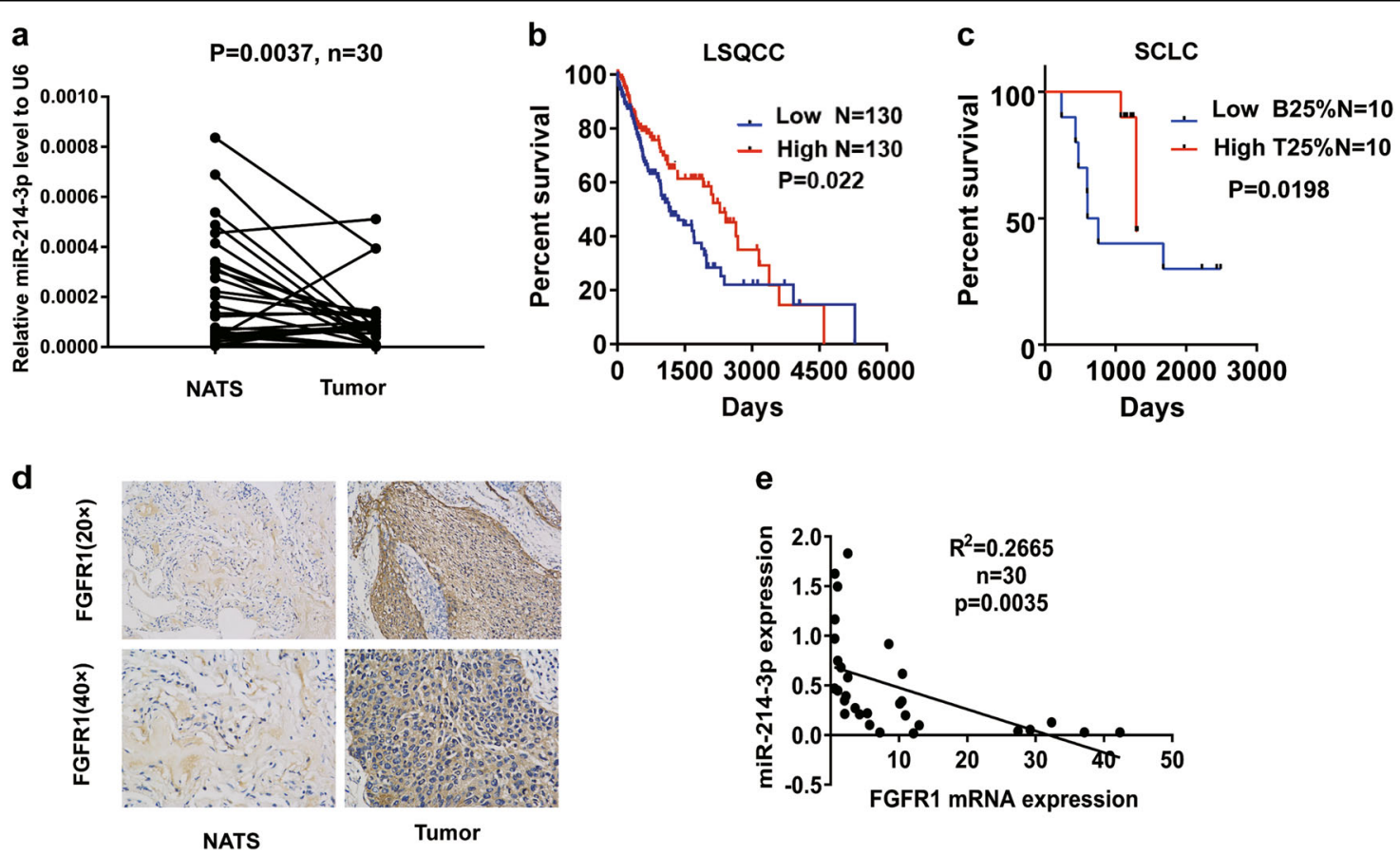

Fig. 1 miR-214-3p expression deregulation correlated with survival rate. a Relative miR-214-3p expression levels was detected by quantitative real-time PCR (qRT-PCR) in lung tumor tissues and non-tumor adjacent tissues (NATs), b Kaplan-Meier curves depicting overall survival (OS) according to the expression of miR-214-3p in lung squamous cell carcinoma (LSQCC) patients in The Cancer Genome Atlas (TCGA) database ( $n=260)$, c Kaplan-Meier curves depicting OS according to the top 25\% and bottom 25\% expression of the miR-214-3p in small-cell lung cancer (SCLC) patients in GSE27435 microRNA (miRNA) profiles, $\mathbf{d}$ the immunohistochemistry (IHC) staining of fibroblast growth factor receptor 1 (FGFR1) in lung tumor tissues and NATs, e linear fit correlation analysis between FGFR1 messenger RNAs (mRNA) and miR-214-3p were conducted in lung cancer patients $(n=30)$. $P$ values were calculated by paired $t$ test

miR-214-3p suppresses oncogenic pathway by targeting FGFR1

After Gene Ontology (GO) data analysis, the pathways of FGFR1 and miR-214-3p related to EMT were explored. The Wnt signaling pathway was identified to be an intersection (Fig. 5a-c). Interestingly, MAPK/AKT (mitogen-activated protein kinase/AKT) signaling pathways as classic oncogenic pathways were regulated by both miR-214-3p and FGFR $1^{32-35}$. Thus, the Wnt/ MAPK/AKT signaling pathways were investigated in this study. Twenty-four hours after transfection of miR-214$3 p$-mimic, the levels of the proteins in Wnt/MAPK/AKT signaling pathways were markedly decreased (Fig. 5d). To further explore the relationship between FGFR1 and the Wnt signaling pathway, the FGFR1 overexpression plasmid and small interfering RNA (siRNA) of FGFR1 were used. FGFR1 overexpression increased the expression of $\beta$-catenin, c-Myc, and cyclinD1, while siRNA of FGFR1 decreased the expression of the above molecules (Fig. 5e). This effect of miR-214-3p on Wnt/MAPK/AKT signaling pathways was offset by FGFR1 overexpression (Fig. 5f). The major function of miRNAs is to regulate their target genes by messenger RNA (mRNA) cleavage or by inhibiting translation, which depend on the suitability with the 3'-UTR of target genes.

The results suggested that miR-214-3p could not only downregulate FGFR1 by post-transcriptional regulation but also inhibit Wnt/MAPK/AKT pathways by suppressing the phosphorylation of FGFR1.

\section{FGFR1 regulates the expression of miR-214-3p through ERK activation}

Interestingly, we found that miR-214-3p was downregulated by FGFR1 inhibitor AZD4547 and upregulated by the exogenous FGFR1 ligand FGF2 (Fig. 6a). To further investigate which signaling pathway downstream of FGFR1 could regulate the expression of miR-214-3p, Wnt pathway inhibitor XAV-939, the AKT pathway inhibitor MK-2206 $2 \mathrm{HCl}$, and the MAPK pathway inhibitor AZD6244 (Fig. S3) were used. MiR-214-3p was only suppressed by AZD6244 (Fig. 6b). AZD6244 could not suppress the level of miR-214-3p in the presence of FGF2 (Fig. 6b). H1581 and DMS114 cells were transfected with the WT extracellular signal-regulated kinase 2 (ERK2) plasmid or the dominant active ERK2_R67S plasmid. Compared with the WT ERK2 and negative control, 
a
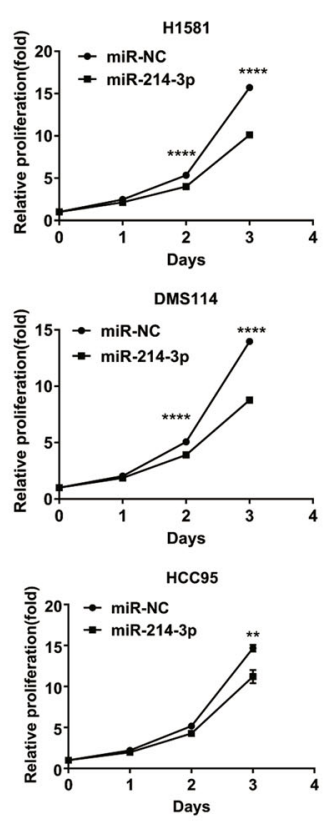

C

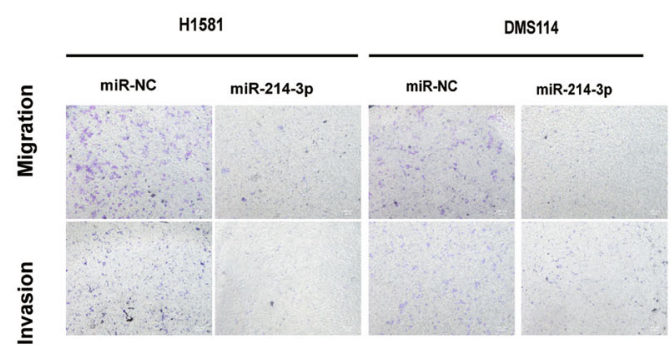

d

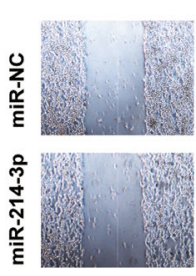

H1581

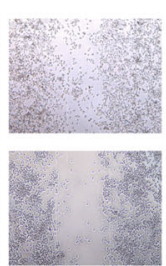

e

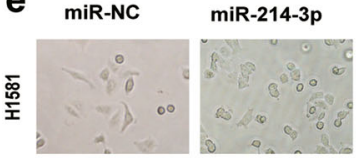

产

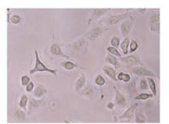

엄

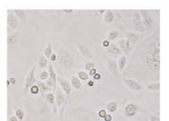

MS114

f b


DMS114
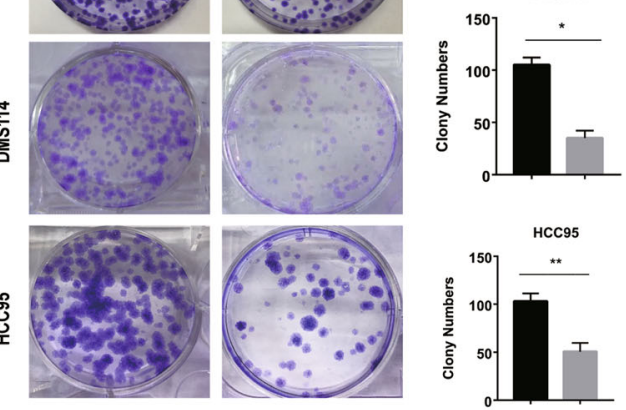

miR-NC
miR-214-3p
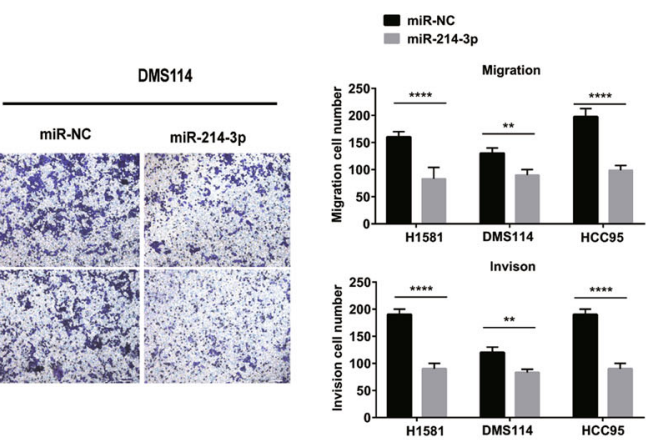

$\mathrm{HCC} 95$


Fig. 2 miR-214-3p suppressed the proliferation, epithelial-mesenchymal transition (EMT) process, and invasion in fibroblast growth factor receptor 1 (FGFR1)-amplified lung cancer cell lines. H1581, DMS114, and HCC95 cell lines were transfected with miR-214-3p mimic or miR-NC. a, b Cell growth was measured by the CCK8 assay and colony assay. c, d Migration and invasion was determined by scratch assay and transwell assay.

e Representative images showing the altered morphological characteristics of the cells. $\mathbf{f}$ Quantification of EMT markers was measured by western blot. $P$ values were calculated by Student's $t$ test: ${ }^{*} p<0.05 ;{ }^{* *} p<0.01 ;{ }^{* * *} p<0.0001$ 


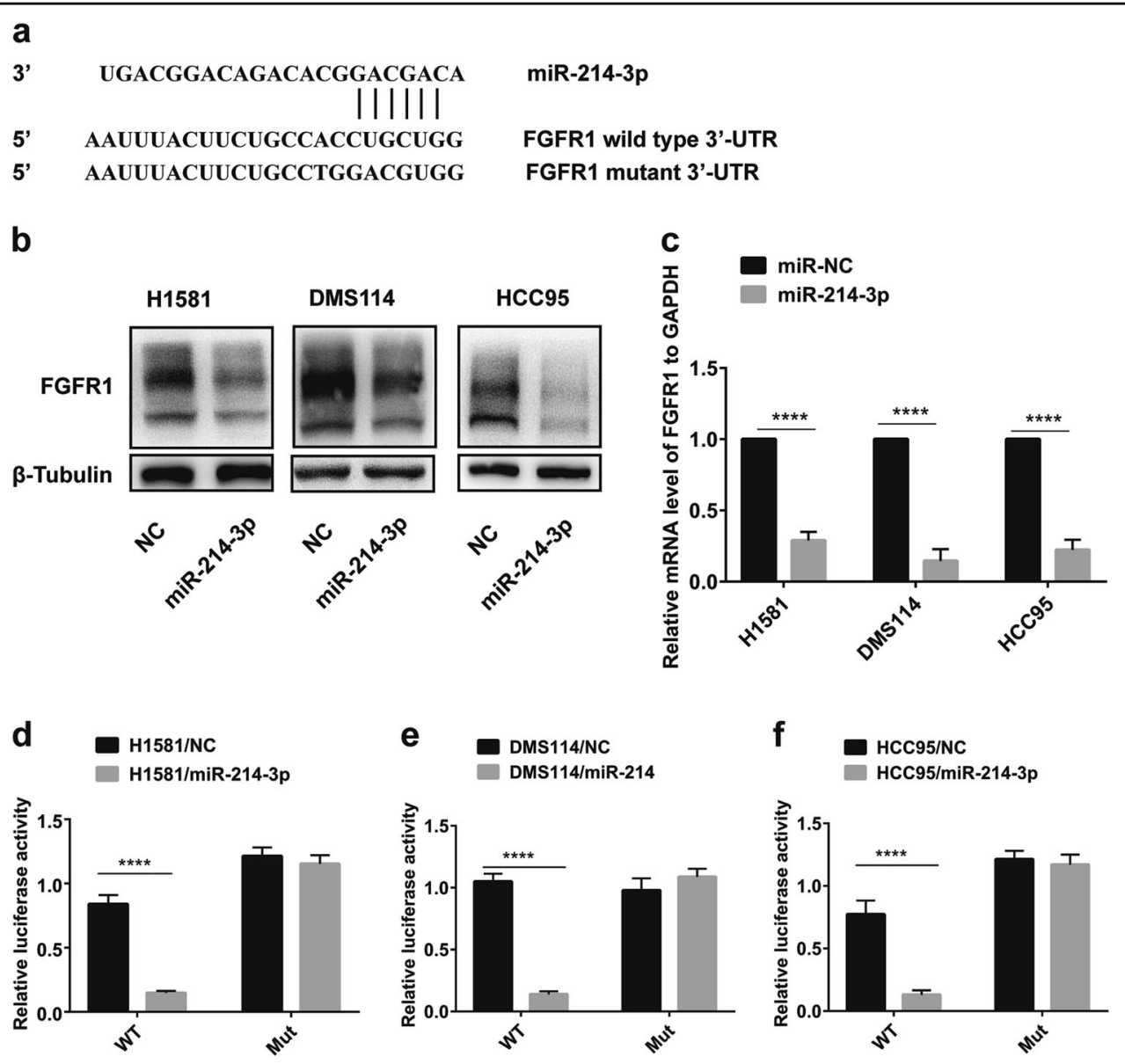

Fig. 3 Fibroblast growth factor receptor 1 (FGFR1) is a direct target of miR-214-3p. a Schematic diagram of the predicted binding site of FGFR1 3'-untranslated region (UTR) and miR-214-3p. b, c Western blot and quantitative real-time PCR (qRT-PCR) analyses of FGFR1 expression levels in H1581 and DMS114 cells transfected with miR-NC or miR-214-3p. d-f Luciferase assay of the reporter plasmids including the wild-type and mutant FGFR1 $3^{\prime}$ UTR. $P$ values were calculated by Student's $t$ test. ${ }^{* * *} p<0.0001$

ERK2_R67S upregulated the level of phosphorylated-ERK, which could not be suppressed by AZD4547 or AZD6244 (Fig. 6c). Meanwhile, the upregulation of miR-214-3p caused by ERK2_R67S plasmid transfection was not suppressed by FGFR1 inhibitor AZD4547 or MEK/ERK inhibitor AZD6244 (Fig. 6d). Therefore, we verified that FGFR1 regulated the expression of miR-214-3p through ERK activation.

\section{miR-214-3p and AZD4547 have synergistic antitumor effects in vitro and in vivo}

Because the addition of the FGFR1 inhibitor AZD4547 actually led to downregulation of the tumor suppressor miR-214-3p in H1581 and DMS114 cells, we hypothesized that the downregulation of miR-214-3p partially accounted for the modest efficacy of AZD4547. To verify this hypothesis, H1581 cells were treated with increasing concentration of AZD4547, ranging from 0.01 to $10 \mu \mathrm{M}$, in combination with miR-214-3p or miR-NC using at a weak, fixed concentration $(0.3 \mathrm{nM})$. The half-maximal inhibitory concentration (IC50) for AZD4547 plus miR$\mathrm{NC}$ was $0.1995 \mu \mathrm{M}$. However, the IC50 for AZD4547 plus miR-214-3p was reduced to $0.01299 \mu \mathrm{M}$. To further explore the interaction of miR-214-3p and AZD4547, we calculated the combination index $(\mathrm{CI})$ between them at different concentrations $(\mathrm{CI}=\mathrm{CA}, x / \mathrm{IC} x, \mathrm{~A}+\mathrm{CB}, \mathrm{x} / \mathrm{IC} x, \mathrm{~B})$. The range of $\mathrm{CI}$ values was below $0.2(\mathrm{CI}<1)$, indicating a strong synergy between miR-214-3p and AZD4547 (Fig. 7a). In the DMS114 cell line, the IC50 for AZD4547 plus miR-NC was $0.361 \mu \mathrm{M}$; however, the IC50 for AZD4547 plus miR-214-3p was decreased to $0.0245 \mu \mathrm{M}$. The range of $\mathrm{CI}$ values was below $0.4(\mathrm{CI}<1)$ (Fig. S4).

To further verify the synergism in vivo, subcutaneous mouse models and orthotopic lung cancer mouse models were established using H1581(Fig. 7b-e) and DMS114 cells (Fig. S4). After a week, miR-214-3p antagomir or 


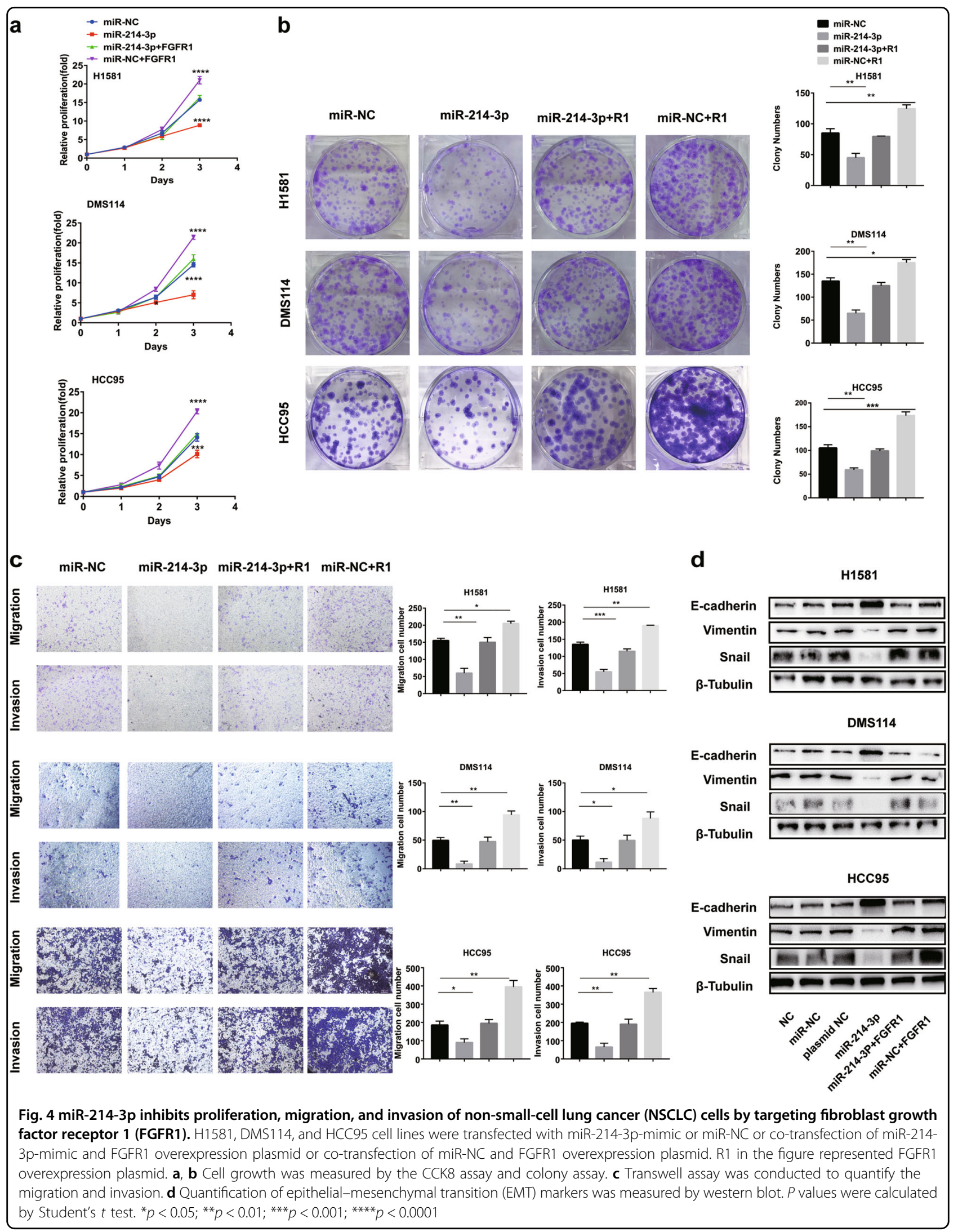


a

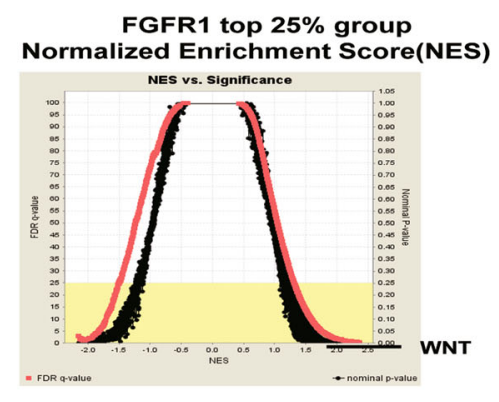

miR-214-3p top 25\% group Normalized Enrichment Score(NES)

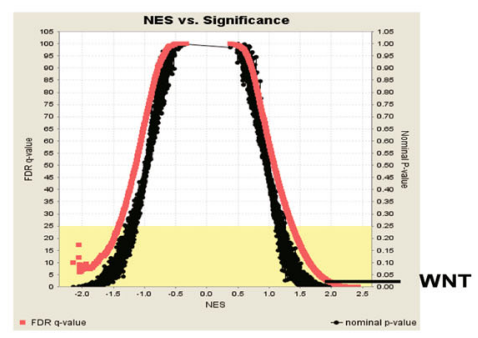

d

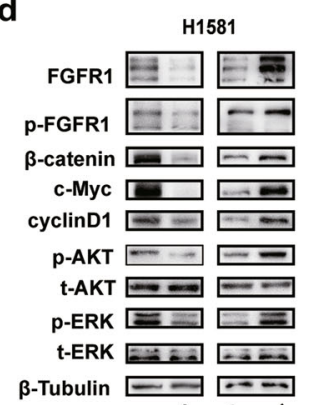

B-Tubulin $=\square$

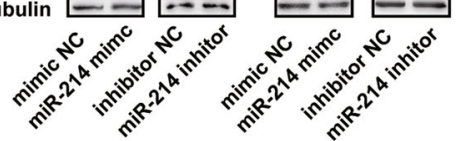

b

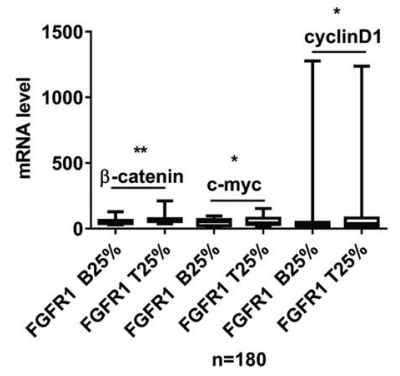

c
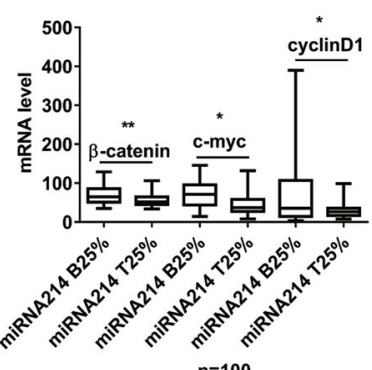

$n=100$

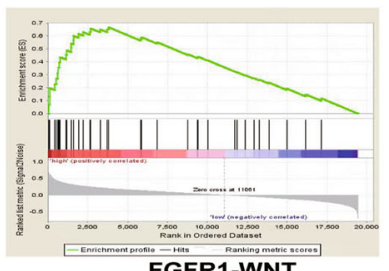

FGFR1-WNT

e

H1581

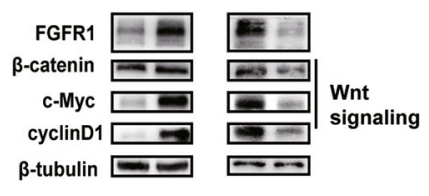

DMS114

FGFR1

-catenin $=0$

c-Myc

cyclinD1 signaling

$\beta$-tubulin

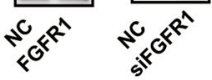

f
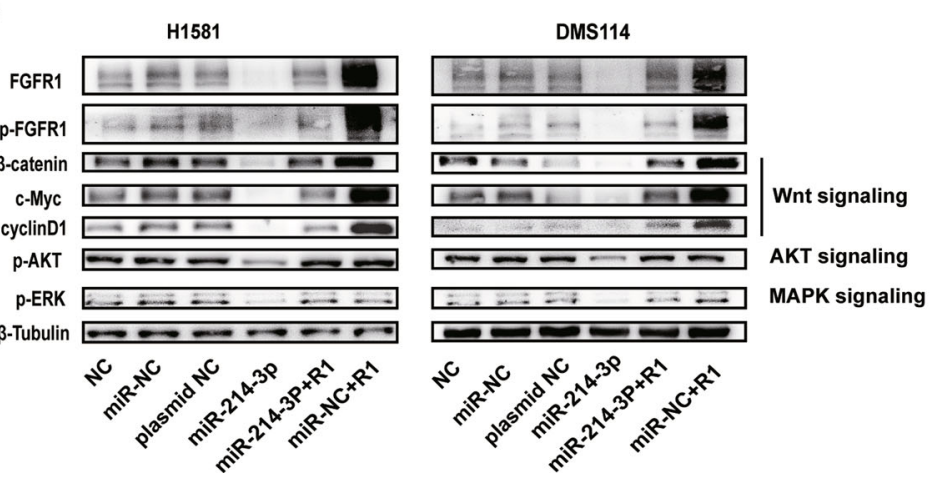

Fig. 5 (See legend on next page.) 
(see figure on previous page)

Fig. 5 miR-214-3p suppresses oncogenic pathway by targeting fibroblast growth factor receptor 1 (FGFR1). a, c A gene set enrichment analysis related to the epithelial-mesenchymal transition (EMT) signatures and hallmarks in the groups with high and low expression of miR-214-3p or FGFR1 in the The Cancer Genome Atlas (TCGA) lung cancer cohort. The signaling pathways were negatively regulated by miR-214-3p and positively regulated by FGFR1. Wnt signaling pathway as an intersection was determined by a normalized enrichment score (NES). $\mathbf{b}$ The mRNA level of $\beta$-catenin, c-Myc, and cyclinD1 in Wnt signaling in the groups with high and low expression of miR-214-3p or FGFR1 in the TCGA lung cancer cohort. $\mathbf{d}$ After transfection of miR-NC and miR-214-3p mimic or anti-miR-NC and anti-miR-214-3p for $24 \mathrm{~h}$, the protein levels of FGFR1, pFGFR1, MAPK (mitogen-activated protein kinase), phosphoinositide 3-kinase-AKT (PI3K-AKT), and Wnt signaling pathway were measured by western blot, and the expression level of FGFR1 were measured by western blot after transfection for $48 \mathrm{~h}$. e After transfection of FGFR1 overexpression plasmid or small interfering RNA (siRNA) of FGFR1 for $48 \mathrm{~h}$, the protein levels of Wnt signaling pathways were measured by western blot. $\mathbf{f}$ After transfection of miR214-3p or FGFR1 plasmid or co-transfection of miR-214-3p and FGFR1 plasmid for $48 \mathrm{~h}$, the protein levels of FGFR1, pFGFR1, MAPK, AKT, and Wnt signaling pathways were measured by western blot. $P$ values were calculated by Student's $t$ test: ${ }^{*} p<0.05 ;{ }^{* *} p<0.01$

miR antagomir NC was injected into the implanted tumor every 3 days for a total of seven injections in subcutaneous mouse models. Tumor volume was measured every 3 days. The results indicated that the subcutaneous tumors in miR-214-3p antagomir group grew slower than the miR-NC antagomir group. Meanwhile, the miR-2143 p antagomir plus AZD4547 group grew even slower than the miR-214-3p antagomir group or the AZD4547 group $(p<0.05$, Fig. 7b).

The orthotopic lung cancer mouse models were established by ad-miR-214-3p virus-transfected cells. The volume of primary lung cancer and the number of metastatic nodules both decreased in an orderly fashion for the following four groups: the LV-miR-NC group, the AZD4547 group, the LV-miR-214-3p group, and the LVmiR-214-3p group plus AZD4547 group $(p<0.05$, Fig. 7c, d). The LV-miR-214-3p group plus AZD4547 group had the longest survival time (Fig. 7e). Hematoxylin and eosin staining was used to quantify the number of nodules in the contralateral lung metastasis (Fig. S4). Immunofluorescence staining for the representative EMT markers were also applied. In the LV-miR-214-3p group plus AZD4547 group, FGFR1 and mesenchymal markers Snail and VIM were significantly suppressed, while the epithelial marker E-cad was highly expressed (Fig. S4).

Overall, we demonstrated the antitumor synergism between miR-214-3p and AZD4547 in vitro and in vivo.

\section{Discussion}

The vital role of miRNAs in tumor initiation and progression has been highlighted in recent studies. MicroRNAs mainly inhibit the processes of post-transcriptional translation as well as the stability of mRNAs, controlling the cellular processes of inflammation, apoptosis, differentiation, migration, cell cycle regulation, and stress response by targeting related genes. We used a panel to identify miRNAs associated with tumor invasion and metastasis in lung cancer patients in this study. Ten miRNAs involved in tumor invasion and metastasis most frequently were detected in tumor tissues vs. NATs $(n=30)^{36-40}$. Among them, miR-214-3p expression was significantly lower in tumor tissues than NATs, which might act as a tumor biomarker. More importantly, miR-214-3p was correlated to a favorable patient prognosis, indicating that miR-214-3p may act as a biomarker to predict the recurrence, and outcome for lung cancer patients.

We subsequently performed a profiling study on the proliferation, invasion, migration, and EMT of FGFR1amplified cell lines, which confirmed the tumorsuppressive effect of miR-214-3p. miR-214 was first reported to be developmentally related, which is subsequently shown to promote the progression and metastasis of melanoma ${ }^{41,42}$. However, previous studies have shown that miR-214-3p could be tumor promoter or suppressor in different tumor types. On the one hand, miR-214-3p increased the resistance to erlotinib and stimulated tumor proliferation and invasion by targeting LHX6 in patients with $\mathrm{LADC}^{43}$. Anti-miR-214 therapy has been shown to inhibit tumor progression in primary breast cancer tumors melanoma and pancreatic neuroendocrine can$\operatorname{cer}^{44}$. On the other hand, miR-214-3p is downregulated and suppresses tumor proliferation and metastasis in other tumors such as cutaneous squamous cell carcinoma and esophageal squamous cell carcinoma ${ }^{45,46}$. These reports indicate that the biological role of miR-214-3p varies up to the genetic background.

Recent studies indicated that miR-214-3p inhibited proliferation or tumor progression by targeting FGFR1 in the differentiation process of osteogenic mesenchymal stem cells and colorectal cancer, which was consistent with our research ${ }^{47,48}$. We confirmed that FGFR1 was a direct target of miR-214-3p and mediated the biological function of miR-214-3p in FGFR1-amplified lung cancer cells.

Wnt signaling mainly regulates development and stemness. The Wnt signaling is commonly divided into independent (non-canonical) signaling and $\beta$-catenindependent (canonical). Wnt signaling is associated with cancer stemness and metastasis. The role of Wnt signaling in carcinogenesis has most prominently been 
a

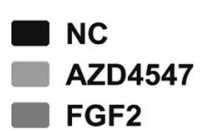

AZD4547+FGF2
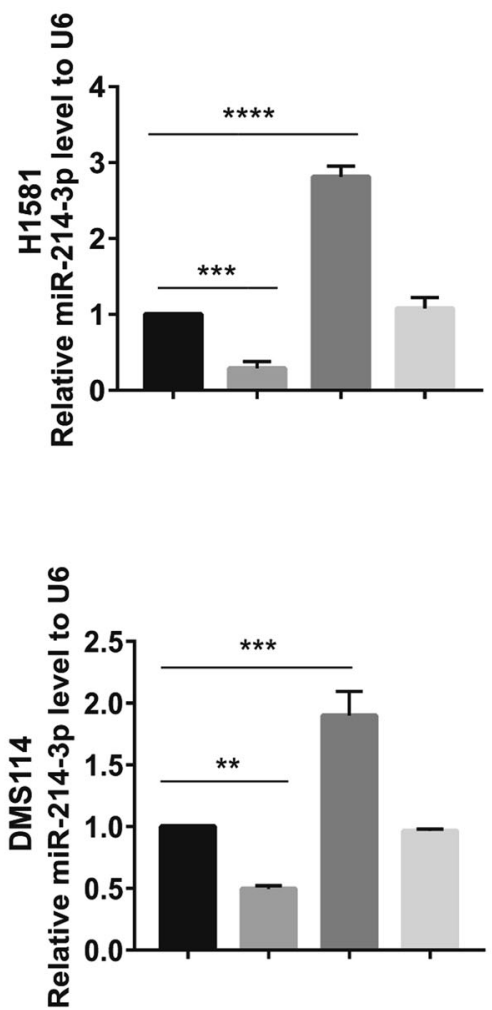

C



b

- NC

AZD6244

FGF2

AZD6244+FGF2
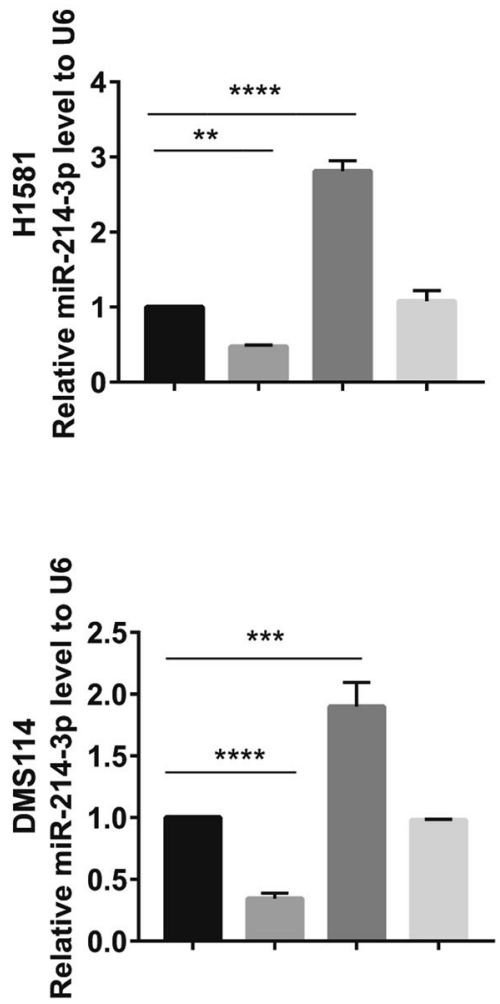

d

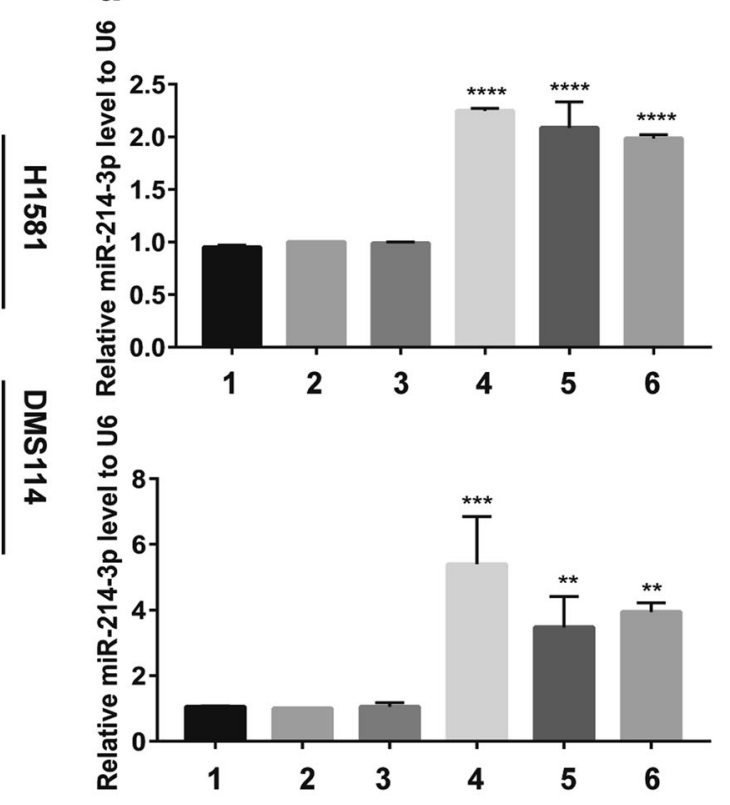

Fig. 6 (See legend on next page.) 
(see figure on previous page)

Fig. 6 Fibroblast growth factor receptor 1 (FGFR1) regulates the expression of miR-214 by regulating extracellular signal-regulated kinase (ERK). a After treatment with FGF2 or FGFR1 inhibitor AZD4547 (1 $\mu \mathrm{M})$ or FGF2 and AZD4547 in H1581 and DMS114 cell lines, the level of miR-214-3p was detected by quantitative real-time PCR (qRT-PCR). b After treatment with MEK/ERK inhibitor AZD6244 (1 $\mu$ M) or FGF2 or FGF2 and AZD6244 $(1 \mu \mathrm{M})$ in $\mathrm{H} 1581$ and DMS114 cell lines, the level of miR-214-3p was detected by qRT-PCR. $\mathbf{c}$, d H1581 and DMS114 cell lines were transfected by plasmid-NC, wild-type ERK2 plasmid (plasmid-ERK2), or autophosphorylation variant ERK2_R67S plasmid (plasmid-ERK_R67S), or plasmid-ERK_R67S followed by treatment of AZD4547 or AZD6244. $\mathbf{c}$ Expression of pERK1/2 and total ERK1/2 were detected by Western blot. d Expression of miR-214-3p were measured by qPCR. $P$ values were calculated by Student's $t$ test: ${ }^{* *} p<0.01 ;{ }^{* *} p<0.001 ;{ }^{* * *} p<0.0001$

described for colorectal cancer, but aberrant Wnt signaling is observed in gastrointestinal cancers, leukemia, melanoma, breast cancer, and so on ${ }^{49}$. To further explore the functional pattern of miR-214-3p in FGFR1-amplified lung cancer, we performed GO data analysis to search the cross-signaling, and the Wnt signaling was found to be an intersection. Previous studies suggest that both miR-214$3 p$ and FGFR1 could regulate MAPK/PI3K-AKT signaling $^{32}$. Thus, we focused on Wnt/MAPK/PI3K-AKT signaling. We confirmed that miR-214-3p inhibited the activity of Wnt/MAPK/PI3K-AKT signaling, and overexpression of FGFR1 offset this effect, which suggest that miR-214-3p inhibited Wnt/MAPK/PI3K-AKT signaling pathway by suppression of FGFR1. Meanwhile, we also demonstrated that the Wnt signaling pathway was regulated by FGFR1. It was well known that Wnt/MAPK/ PI3K-AKT signaling pathway promoted the progression of EMT in lung cancer, prostate cancer, nasopharyngeal carcinoma, cervical cancer, breast cancer, and so on ${ }^{50-54}$. Therefore, we, for the first time, proposed that existence of miR-214-3p-FGFR1-Wnt/MAPK/PI3K-AKT axis in FGFR1-amplified lung cancer.

It has long been recognized that FGFRs are overexpressed in many types of cancer cells, including NSCLC, breast cancer, and oral SQCC ${ }^{35,55-57}$. As mentioned above, FGFR1 as an attractive candidate target showed modest response. Therefore, genes' co-alternation with FGFR1 or signaling paradigm have been widely studied, such as the co-active receptor tyrosine kinases, the co-activation of MTOR (mechanistic target of rapamycin) pathway, and so on. ${ }^{58,59}$ Our previous studies also confirmed that FGFR1 co-expressed with Gli2, SOX2, and YAP to maintain stemness or EMT in lung cancer ${ }^{21,60}$. Here, we confirmed the antitumor synergism between miR-214-3p and AZD4547 in vitro and in vivo. In the cell experiment, the $\mathrm{CI}$ index is about 0.2 , showing a strong synergistic effect. Furthermore, subcutaneous and orthotopic lung cancer models also confirmed the synergistic effect.

The underlying molecular mechanism of the synergy between miR-214-3p and AZD4547 has remained unknown. Interestingly, we found a loop in which FGFR1 can simultaneously regulate the level of miR-214-3p through ERK. FGFR1 inhibitor AZD4547 reduced the level of miR-214-3p by suppressing ERK activation in cell lines. However, it was confirmed that miR-214-3p acted as a tumor suppressor by targeting FGFR1. Therefore, FGFR1 inhibitor AZD4547 might upregulate the level of FGFR1 and the pathway downstream of FGFR1 by inhibiting miR214-3p. Meanwhile, we did notice that a dynamic balance between miR-214-3p and FGFR1 existed within $96 \mathrm{~h}$ after the addition of AZD4547, which need further exploration. Previous studies identified DUSP6 deletion and NRAS amplification in drug-resistant $\mathrm{H} 1581$ cells, leading to the reactivation of MAPK pathway. Meanwhile, drug-resistant DMS114 cells drove MAPK pathway reactivation by suppressing upregulation of $\mathrm{MET}^{61}$. Therefore, in future studies it is critical to investigate the relationship between DUSP6, NRAS, miR-214-3p, and MAPK signaling in drugresistant H1581 cells, and the correlation of MET, miR-214$3 p$, and MAPK signaling in drug-resistant DMS114 cells.

The microRNA mimic has been discovered and employed for cancer therapy in the clinical setting since 2013. In addition, clinical trials of microRNAs for patient prognosis and treatment are currently underway ${ }^{62}$. As stated above, our findings in this study have demonstrated that miR-214-3p acts as a vital biological targeting inhibitor for FGFR1, which need further study for cancer treatment.

In summary, in this study we have discovered a negative feedback regulatory axis between miR-214-3p and FGFR1, as well as a synergistic antitumor effect between miR-214$3 p$ and AZD4547. These findings may provide new insights for the prognosis and treatment of patients with FGFR1-amplified lung cancer.

\section{Materials and methods Cells and reagents}

H1581, DMS114, and HCC95 were purchased from the American Type Culture Collection and were authenticated by STR profiling (Table. S1) ${ }^{21}$. The cell lines were cultured with RPMI-1640 (HyClone) with 10\% fetal bovine serum (FBS) (Gibco). FGFR1 inhibitor AZD4547 was kindly provided by AstraZeneca Pharmaceutical Company $^{63}$. AZD6244 was obtained from Selleck Chemicals ${ }^{64}$. FGF2 was purchased from PeproTech. 
a
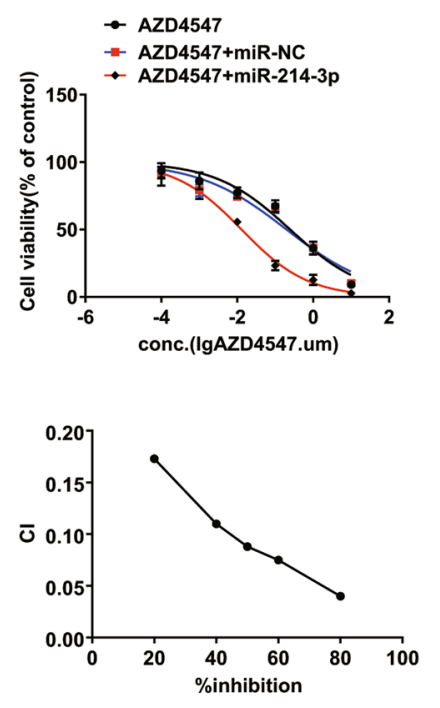

C

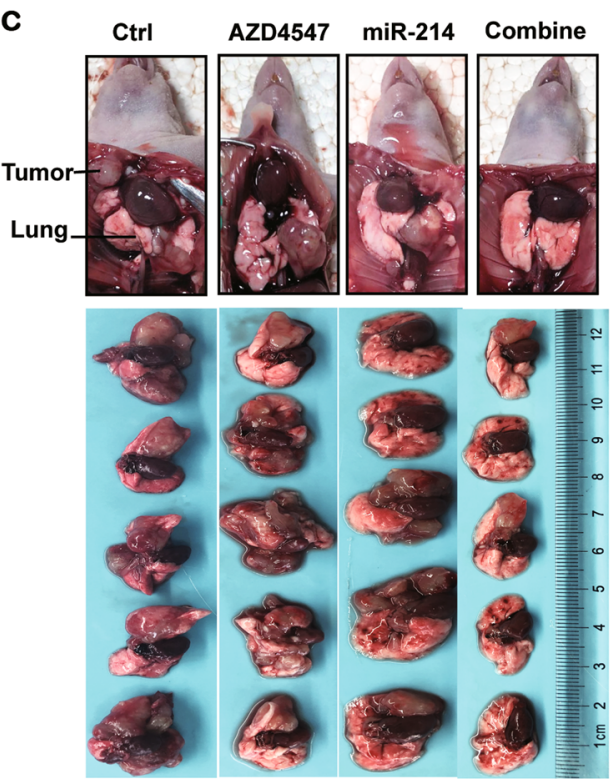

- NC

miR-214-3p

AZD4547

AZD4547+miR-214-3p



\section{b}

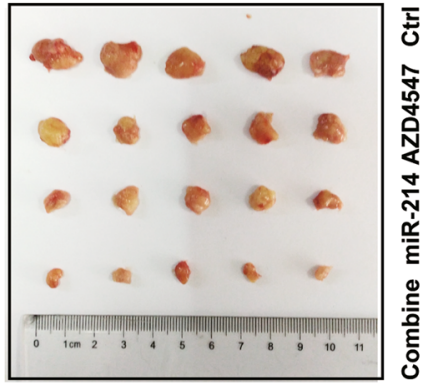

$\rightarrow$ NC

- AZD4547+miR214 agomir NC

- miR214 agomir



d
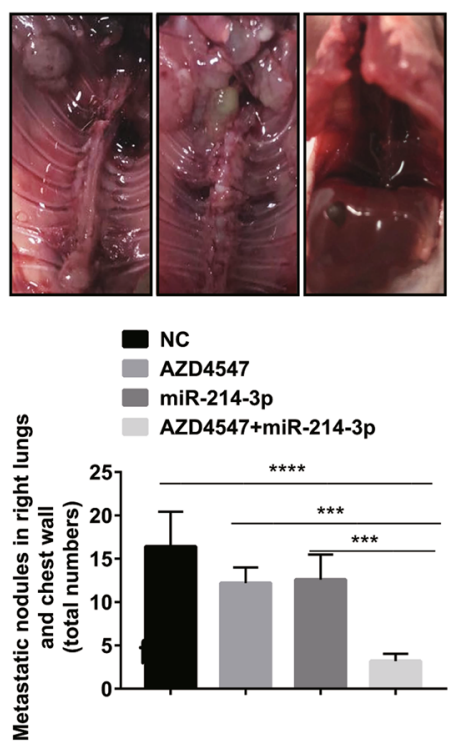

e

$$
\begin{aligned}
& \text { - NC } \\
& + \text { AZD4547 } \\
& \text { - miR-214-3p } \\
& \text { - AZD4547+miR-214-3p }
\end{aligned}
$$

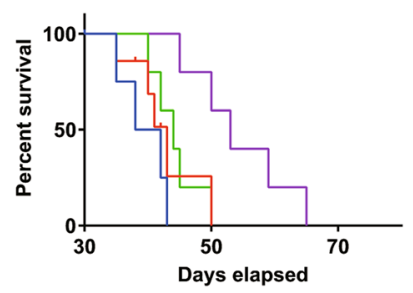

Fig. 7 (See legend on next page.) 
(see figure on previous page)

Fig. 7 miR-214 and AZD4547 have synergistic effects in vitro and in vivo. The experiments were conduct using H1581 (this figure) and DMS114 cells (Fig. S4). a H1581 cells were transfected with $0.3 \mathrm{nM}$ miR-NC or miR-214-3p, and then incubated with AZD4547 in a serial dilution. After $72 \mathrm{~h}$, CCK8 was used to evaluate cellular proliferation. $\mathrm{Cl}$ values were determined by non-linear regression methods at any given effect. (Cl=1, additivity; Cl.1, antagonism; $\mathrm{Cl}, 1$, synergy). b Representative images showing tumor formation in the nude mices treated with microRNA (miRNA) agomir NC, AZD4547, miR-214-3p agomir or miR-214-3p, and agomir for 3 weeks (combined). c-e H1581 ( $2 \times 10^{6}$ cells) transfected with LV-miR-214-3p or LV-NC in a volume of $50 \mu \mathrm{L}$ (phosphate-buffered slaine (PBS):Matrigel = 4:1) were injected into the left lung of 6-week-old male BCLB/C nude mice $(n=20)$. One week after injection, five mice injected with LV-miR-214-3p and five mice injected with LV-NC were treated with FGFR1 inhibitor AZD4547 $(12.5 \mathrm{mg} / \mathrm{kg} / \mathrm{day})$ randomly for 3 weeks. c Representative images showing tumor formation in the nude mice treated with LV-NC, AZD4547, LV-miR214-3p, or LV-miR-214-3p combined with AZD4547 for 3 weeks. d Metastatic tumors in right lung and chest wall. e Survival curve for the mice in each treatment group evaluated. $P$ values were calculated by Student's $t$ test: ${ }^{* *} p<0.001 ;{ }^{* * *} p<0.0001$

miRNA, siRNA transfection, and plasmid construction

The miRNA mimics, inhibitors, and antagomir were obtained from RiboBio (Guangzhou, China). miRNA NC, miRNA mimics, and miRNA inhibitors were transfected by Lipofectamine 3000 (Invitrogen, Carlsbad, USA). The ad-miR-214-3p virus was purchased from Hanbio Biotechnology Co. Ltd. (Shanghai, China). The FGFR1 overexpression plasmid was purchased from biolink (Shanghai, China). The sequences of siFGFR1, siRNA-NC, WT ERK2, and autophosphorylation variant ERK2 p.R67S (ERK2_R67S) were described by us before ${ }^{21}$.

\section{Colony formation assay}

Cells were transfected into miR-214-3p mimics and inhibitors. After $24 \mathrm{~h}$, cells were digested with trypsin and replanted at a density of 1000 cells $/ 10 \mathrm{~cm}^{2}$. After 3 weeks of incubation, cells were fixed with methanol and then stained with $0.1 \%$ crystal violet. Colonies larger than $100 \mu \mathrm{m}$ in diameter were counted, and each assay was repeated in triple.

\section{Quantitative real-time PCR}

Lung cancer tissues NATs) were provided by Shanghai Chest Hospital, Jiao Tong University (Shanghai, China). Written, informed consents approving the use of tissue samples for research purposes were obtained from lung cancer patients. The study was approved by the Institute Research Ethics Committee of Shanghai Jiao Tong University $^{60}$. Total RNA was isolated from the above cells or frozen tissues using Trizol reagent (Invitrogen). Complementary DNA was prepared with 2 ng of total RNA for miRNA and mRNA quantification using a Mir-X miRNA First-Strand Synthesis Kit (Clontech Laboratories Inc., USA) and PrimeScript ${ }^{\mathrm{TM}}$ RT Master Mix Kit (Takara, Dalian, China). RNA expression was measured by qRT-PCR using a Mir-X miRNA qRT-PCR SYBR Kit (Clontech Laboratories Inc., USA) and a SYBR ${ }^{\circledast}$ Green (Takara, Dalian, China), according to the manufacturer's protocol. U6 or GAPDH (glyceraldehyde 3-phosphate dehydrogenase) was used for the normalization. The primers for miRNA and U6 were purchased from RiboBio Company (Guangzhou, China).
The sequences of the primers are covered by a patent. The primer sequences of this study were as follows:

ZO-1 (forward, 5' -AGCGAAGCCACCTGAAGATA-3' and reverse, 5'-GATGGCCAGCAGGAATATGT-3').

Snail (forward, 5'-CGCGCTCTTTCCTCGTCAG-3' and reverse, 5'-TCCCAGATGAGCATTGGCAG-3'), the other primer sequences were described by us before ${ }^{21}$.

\section{Scratch assay and Transwell migration/invasion assay}

Scratch assay was described by us before ${ }^{21}$. Cells $(5 \times$ $10^{4}$ ) with $1 \%$ FBS medium were seeded in an $8-\mu \mathrm{m}$ pore membrane with Matrigel-coated or not (Corning, 356231), and 10\% FBS medium was placed in the lower chamber. After $36 \mathrm{~h}$ incubation, cells on the upper layer were removed using a cotton swab. Passing through cells were fixed in methanol for $20 \mathrm{~min}$ and then stained for 20 min with $0.1 \%$ crystal violet dye. The stained cells were counted at three randomly selected views for subsequent calculations.

\section{CCK8 assay}

CCK-8 assay kit (Dojindo Laboratories, Kumamoto, Japan) was used to examine the proliferation rate under treatment. Briefly, $2 \times 10^{3}$ cells were seeded into 96-well plates. After transfected with miR-214-3p mimic or miR214-3p inhibitor for $24 \mathrm{~h}, 10 \mu \mathrm{L}$ CCK 8 reagent was added into the medium per well. The absorbance at $450 \mathrm{~nm}$ was measured by the microplate reader (Synergy2, BioTek, Winooski, VT) after 1 to $3 \mathrm{~h}$ coincubation.

\section{Western blot}

Treated cells or tissues pulverized by ultrasonic waves were lysed by RIPA containing the Complete Protease Inhibitor Cocktail. Phosphatase Inhibitor Cocktail and phenylmethylsulfonyl fluoride. After centrifugation at 12,000 r.p.m. for $20 \mathrm{~min}$ at $4{ }^{\circ} \mathrm{C}$, the supernatant was procured for the next step. An equal amount of $30 \mu \mathrm{g}$ protein was separated by $10 \%$ sodium dodecyl sulfatepolyacrylamide gel electrophoresis gel and then transferred onto NC membranes (Millipore, Billerica, MA, USA). After blocking with $5 \%$ bovine serum albumin for 
$1 \mathrm{~h}$, antibodies were incubated overnight at $4{ }^{\circ} \mathrm{C}$. The intensity of the signal was detected by the ECL chemical image $^{21}$. All the antibodies were purchased from Cell Signaling Technology (Beverly, MA, USA).

\section{Immunofluorescence microscopy}

The tissue sections were incubated with primary antibody overnight after being rehydrated. The secondary antibody was donkey anti-rabbit immunoglobulin G conjugated with Alexa Fluor 594. Cell nuclei were stained with 4',6-diamidino-2-phenylindole (Sigma) Stained cells were photographed and quantified under an immunofluorescence microscope (Leica DFC420C).

\section{Immunohistochemistry}

After rehydrating, tissue specimens were incubated with primary antibodies for $1 \mathrm{~h}$, followed by incubation of the secondary antibodies. Diaminobenzidine-hydrogen peroxide (Sigma) was the chromogen, and the counterstaining was carried out with $0.5 \%$ hematoxylin. The degree of IHC was classified into the following grades (-, no staining;,$+ 10 \%$;,$++ 10-50 \%$; and,$+++>50 \%$ )

In vivo xenograft assay and orthotropic lung tumor model

In the subcutaneous xenograft model, H1581 $\left(1 \times 10^{6}\right.$ cells) or DMS114 $\left(2 \times 10^{6}\right.$ cells $)$ were injected into the right side of the nude mouse in a volume of $50 \mu \mathrm{L}$. AZD4547 $(12.5 \mathrm{mg} / \mathrm{kg} /$ day $)$ was given to nude mice by gavage for 3 weeks. In the orthotopic xenograft model, H1581 $\left(2 \times 10^{6}\right.$ cells $)$ or DMS114 $\left(3 \times 10^{6}\right.$ cells $)$ cell suspensions in a volume of $50 \mu \mathrm{L}$ were injected through the intercostal space into the left lung of nude mice. AZD4547 $(12.5 \mathrm{mg} / \mathrm{kg} /$ day $)$ was given by gavage for 2 weeks. All the nude mice were housed in the SPF (specific pathogen free) animal room of Shanghai Jiao Tong University. All animal experiments were carried out in accordance with the approved scheme of the Shanghai Jiao Tong University Institutional Ethics Committee.

\section{Luciferase reporter assays and transient transfection}

The 3'-UTR sequence of FGFR1 interacting with miR214 and the corresponding mutant sequence within the predicted target site were predicted to be synthesized and inserted into the pmiR-GLO dual-luciferase miRNA target expression vector (Guangdong Ruibo Biotech Co. Ltd.) called WT-FGFR1 3'-UTR and mt-FGFR1 3'- UTR. Subsequently, H1581 or DMS114 cells were plated into 24well plates and transfected with these vectors. Cells were co-transfected with miR-214 and WT-FGFR1 3'-UTR, miR-214 and MT-FGFR1 $3{ }^{\prime}$-UTR, miR-NC and WTFGFR1 3'-UTR, and miR-NC and mt-FGFR1 3'-UTR. After $48 \mathrm{~h}$, cells were measured according to the procedure of manufacturer's instructions (Dual-Luciferase Assay
System; Promega). pRL-TK expressing Renilla luciferase is co-transfected to use as an internal reference.

\section{Analysis of public datasets from TCGA}

Prognostic values of miR-214-3p level were downloaded from the TCGA database and analyzed by Kaplan-Meier survival curves of NSCLC patients, using Kaplan-Meier plotter (www.kmplot.com/analysis).

\section{Statistical analysis}

All the data were presented as mean \pm SEM or SD and analyzed using the GraphPad Prism software (version7.0a, GraphPad Software Inc.). All experiments have three independent operations. We used Student's $t$ tests to compare changes in miRNA levels between tumor tissues and corresponding paired normal tissues. $P$ value $<0.05$ was considered statistically significant.

\section{Acknowledgements}

This work was funded by the National Key R\&D Program of China (2016YFC1303300 to S.L), Shanghai Chest Hospital Project of Collaborative Innovation (YJXT20190105, YJXT20190209, to S.L and Z.L.), Clinical Research Plan of SHDC (16CR3005A to S. L.), Shanghai Science and Technology Commission guidance projects (18411968200 to Z.L.), Medical-Engineering Joint Funds of Shanghai Jiao Tong University YG2017MS81 (to Z.L.), Shanghai Youth Top Talent Project (to Z.L.), the National Natural Science Foundation of China (81672272 to S.L. and 81773115 to W.X.), the National Key Grant of China (2016YFC0906400 to W.X.), the Key project of Shanghai Health and Family Planning (201540365 to S.L.), Shanghai Municipal Science and Technology Commission Research Project (17431906103 to S.L.), and Shanghai Scientific Research Projects (14140902800 to S.L.). We thank AstraZeneca Pharmaceutical Company for kindly supply of AZD4547 and for AstraZeneca Research Funding.

\section{Conflict of interest}

The authors declare that they have no conflict of interest.

\section{Publisher's note}

Springer Nature remains neutral with regard to jurisdictional claims in published maps and institutional affiliations.

Supplementary Information accompanies this paper at (https://doi.org/ 10.1038/s41389-019-0151-1).

Received: 17 February 2019 Revised: 11 April 2019 Accepted: 21 May 2019 Published online: 06 September 2019

\section{References}

1. Siegel, R. L., Miller, K. D. \& Jemal, A. Cancer statistics, 2018. CA Cancer J. Clin. 68, 7-30 (2018).

2. Bray, F. et al. Global cancer statistics 2018: GLOBOCAN estimates of incidence and mortality worldwide for 36 cancers in 185 countries. CA Cancer J. Clin. 68, 394-424 (2018).

3. Houston, K. A., Mitchell, K. A., King, J., White, A. \& Ryan, B. M. Histologic lung cancer incidence rates and trends vary by race/ethnicity and residential county. J. Thorac. Oncol. 13, 497-509 (2018).

4. Lewis, D. R., Check, D. P., Caporaso, N. E., Travis, W. D. \& Devesa, S. S. US lung cancer trends by histologic type. Cancer 120, 2883-2892 (2014).

5. Polyak, K. \& Weinberg, R. A. Transitions between epithelial and mesenchymal states: acquisition of malignant and stem cell traits. Nat. Rev. Cancer $\mathbf{9}$, 265-273 (2009) 
6. Cancer Genome Atlas Research N. Comprehensive molecular profiling of lung adenocarcinoma. Nature 511, 543-550 (2014).

7. Cancer Genome Atlas Research N. Comprehensive genomic characterization of squamous cell lung cancers. Nature 489, 519-525 (2012).

8. Helsten, T. et al. The FGFR landscape in cancer: analysis of 4,853 tumors by next-generation sequencing. Clin. Cancer Res. 22, 259-267 (2016).

9. Kim, H. R. et al. Fibroblast growth factor receptor 1 gene amplification is associated with poor survival and cigarette smoking dosage in patients with resected squamous cell lung cancer. J. Clin. Oncol. 31, 731-737 (2013).

10. Schultheis, A. M. et al. Fibroblast growth factor receptor 1 (FGFR1) amplification is a potential therapeutic target in small-cell lung cancer. Mod. Pathol. 27, 214-221 (2014).

11. Sousa, V. et al. Amplification of FGFR1 gene and expression of FGFR1 protein is found in different histological types of lung carcinoma. Virchows Arch. 469, 173-182 (2016).

12. Gilbert, J. A. BGJ398 for FGFR-altered advanced cholangiocarcinoma. Lancet Oncol. 19, e16 (2018). https://doi.org/10.1016/S1470-2045(17)30902-6.

13. Kelly C. M. et al. A phase lb study of BGJ398, a pan-FGFR kinase inhibitor in combination with imatinib in patients with advanced gastrointestinal stromal tumor. Invest. New Drugs 37, 282-290 (2018). https://doi.org/10.1007/s10637018-0648-z.

14. Paik, P. K. et al. A phase Ib open-label multicenter study of AZD4547 in patients with advanced squamous cell lung cancers. Clin. Cancer Res. 23 5366-5373 (2017).

15. Pal, S. K. et al. Efficacy of BGJ398, a fibroblast growth factor receptor 1-3 inhibitor, in patients with previously treated advanced urothelial carcinoma with FGFR3 alterations. Cancer Discov. 8, 812-821 (2018).

16. Ryan, M. R., Sohl, C. D., Luo, B. \& Anderson, K. S. The FGFR1 V561M gatekeeper mutation drives AZD4547 resistance through STAT3 activation and EMT. Mol. Cancer Res. 17, 532-543 (2018). https://doi.org/10.1158/1541-7786. MCR-18-0429.

17. Michael, M. et al. A phase 1 study of LY2874455, an oral selective pan-FGFR Inhibitor, in patients with advanced cancer. Target. Oncol. 12, 463-474 (2017).

18. Wu D. et al. LY2874455 potently inhibits FGFR gatekeeper mutants and overcomes mutation-based resistance. Chem. Commun. (Camb), 54, 12089-12092 (2018). https://doi.org/10.1039/c8cc07546h.

19. Dutt, A. et al. Inhibitor-sensitive FGFR1 amplification in human non-small cell lung cancer. PLOS ONE 6, e20351 (2011).

20. Weiss, J. et al. Frequent and focal FGFR1 amplification associates with therapeutically tractable FGFR1 dependency in squamous cell lung cancer. Sci. Transl. Med. 2, 62ra93 (2010).

21. Wang, K. et al. FGFR1-ERK1/2-SOX2 axis promotes cell proliferation, epithelial-mesenchymal transition, and metastasis in FGFR1-amplified lung cancer. Oncogene 37, 5340-5354 (2018). https://doi.org/10.1038/ s41388-018-0311-3.

22. Acevedo, V. D. et al. Inducible FGFR-1 activation leads to irreversible prostate adenocarcinoma and an epithelial-to-mesenchymal transition. Cancer Cell 12, 559-571 (2007).

23. Nguyen, P. T. et al. The FGFR1 inhibitor PD173074 induces mesenchymalepithelial transition through the transcription factor AP-1. Br. J. Cancer 109, 2248-2258 (2013).

24. Yang, M. et al. TNFAIP3 is required for FGFR1 activation-promoted proliferation and tumorigenesis of premalignant DCIS.COM human mammary epithelial cells. Breast Cancer Res. 20, 97 (2018).

25. Shibue, T. \& Weinberg, R. A. EMT, CSCS, and drug resistance: the mechanistic link and clinical implications. Nat. Rev. Clin. Oncol. 14, 611-629 (2017).

26. Shi, L. et al. miR-127 promotes EMT and stem-like traits in lung cancer through a feed-forward regulatory loop. Oncogene 36, 1631-1643 (2017).

27. Wang, L. et al. miR-296 inhibits proliferation and induces apoptosis by targeting FGFR1 in human hepatocellular carcinoma. FEBS Lett. 590, 4252-4262 (2016).

28. Xu, Q. et al. MicroRNA-1296 inhibits metastasis and epithelial-mesenchymal transition of hepatocellular carcinoma by targeting SRPK1-mediated PI3K/AKT pathway. Mol. Cancer 16, 103 (2017).

29. Ye, Y. Y. et al. MicroRNA-30a-5p inhibits gallbladder cancer cell proliferation, migration and metastasis by targeting E2F7. Cell Death Dis. 9, 410 (2018).

30. Alvarez-Garcia, I. \& Miska, E. A. MicroRNA functions in animal development and human disease. Development 132, 4653-4662 (2005).

31. Bartel, D. P. MicroRNAs: genomics, biogenesis, mechanism, and function. Cell 116, 281-297 (2004).
32. Cai, H., Miao, M. \& Wang, Z. miR-214-3p promotes the proliferation, migration and invasion of osteosarcoma cells by targeting CADM1. Oncol. Lett. 16, 2620-2628 (2018).

33. Qi, W. et al. Targeting the Wnt-regulatory protein CTNNBIP1 by microRNA-214 enhances the stemness and self-renewal of cancer stem-like cells in lung adenocarcinomas. Stem Cells 33, 3423-3436 (2015).

34. Zhao, C. et al. miR-214 promotes osteoclastogenesis by targeting Pten/PI3K Akt pathway. RNA Biol. 12, 343-353 (2015).

35. Turner, N. \& Grose, R. Fibroblast growth factor signalling: from development to cancer. Nat. Rev. Cancer 10, 116-129 (2010).

36. Bar-Eli, M. Searching for the "melano-miRs": miR-214 drives melanoma metastasis. EMBO J. 30, 1880-1881 (2011).

37. Baumgartner, $U$. et al. miR-19b enhances proliferation and apoptosis resistance via the EGFR signaling pathway by targeting PP2A and BIM in non-small cell lung cancer. Mol. Cancer 17, 44 (2018).

38. Huang, G., Nishimoto, K., Zhou, Z., Hughes, D. \& Kleinerman, E. S. miR-20a encoded by the miR-17-92 cluster increases the metastatic potential of osteosarcoma cells by regulating Fas expression. Cancer Res. 72, 908-916 (2012).

39. Lin, H. Y., Chiang, C. H. \& Hung, W. C. STAT3 upregulates miR-92a to inhibit RECK expression and to promote invasiveness of lung cancer cells. Br. J. Cancer 109, 731-738 (2013).

40. Zaravinos, A. The regulatory role of microRNAs in EMT and cancer. J. Oncol. 2015, 865816 (2015).

41. Flynt, A. S., Li, N., Thatcher, E. J., Solnica-Krezel, L. \& Patton, J. G. Zebrafish miR214 modulates Hedgehog signaling to specify muscle cell fate. Nat. Genet. 39, 259-263 (2007)

42. Penna, E. et al. microRNA-214 contributes to melanoma tumour progression through suppression of TFAP2C. EMBO J. 30, 1990-2007 (2011).

43. Liao, J. et al. Down-regulation of miR-214 reverses erlotinib resistance in nonsmall-cell lung cancer through up-regulating LHX6 expression. Sci. Rep. 7, 781 (2017).

44. Dettori, D. et al. Therapeutic ilencing of miR-214 inhibits tumor progression in multiple mouse models. Mol. Ther. 26, 2008-2018 (2018).

45. Phatak, P. et al. Overexpression of miR-214-3p in esophageal squamous cancer cells enhances sensitivity to cisplatin by targeting survivin directly and indirectly through CUG-BP1. Oncogene 35, 2087-2097 (2016).

46. Yamane, K. et al. Down-regulation of miR-124/-214 in cutaneous squamous cell carcinoma mediates abnormal cell proliferation via the induction of ERK. J. Mol. Med. (Berl.) 91, 69-81 (2013).

47. D, C., Z, qW., Z, Z. \& Wu, W. J. et al. Identification of MicroRNA-214 as a negative regulator of colorectal cancer liver metastasis by way of regulation of fibroblast growth factor receptor 1 expression. Hepatology 60, 598-609 (2014).

48. Yang, L. et al. MiR-214 attenuates osteogenic differentiation of mesenchymal stem cells via targeting FGFR1. Cell Physiol. Biochem. 38, 809-820 (2016).

49. Zhan, T., Rindtorff, N. \& Boutros, M. Wht signaling in cancer. Oncogene $\mathbf{3 6}$, 1461-1473 (2017)

50. Chen, Z. et al. Mn12Ac inhibits the migration, invasion and epithelialmesenchymal transition of lung cancer cells by downregulating the Wnt/ beta-catenin and PI3K/AKT signaling pathways. Oncol. Lett. 16, 3943-3948 (2018).

51. Cheng, Y. FEZF1-AS1 is a key regulator of cell cycle, epithelial-mesenchymal transition and Wnt/beta-catenin signaling in nasopharyngeal carcinoma cells. Biosci. Rep. 39, (2018). https://doi.org/10.1042/BSR20180906.

52. Gao, J. et al. MicroRNA-590-5p functions as a tumor suppressor in breast cancer conferring inhibitory effects on cell migration, invasion, and epithelialmesenchymal transition by downregulating the Wnt-beta-catenin signaling pathway. J. Cell Physiol. 234, 1827-1841 (2018).

53. Guo, X. et al. Long noncoding RNA HOTAIR knockdown inhibits autophagy and epithelial-mesenchymal transition through the Wnt signaling pathway in radioresistant human cervical cancer HeLa cells. J. Cell Physiol. 234, 3478-3489 (2018).

54. Wu, S. et al. 2'Hydroxyflavanone inhibits epithelialmesenchymal transition, and cell migration and invasion via suppression of the Wnt/betacatenin signaling pathway in prostate cancer. Oncol. Rep. 40, 2836-2843 (2018).

55. Freier, $\mathrm{K}$. et al. Recurrent FGFR1 amplification and high FGFR1 protein expression in oral squamous cell carcinoma (OSCC). Oral. Oncol. 43, 60-66 (2007).

56. Reis-Filho, J. S. et al. FGFR1 emerges as a potential therapeutic target for lobular breast carcinomas. Clin. Cancer Res. 12, 6652-6662 (2006). 
57. Turner, N. et al. FGFR1 amplification drives endocrine therapy resistance and is a therapeutic target in breast cancer. Cancer Res. 70, 2085-2094 (2010).

58. Kotani, H. et al. Co-active receptor tyrosine kinases mitigate the effect of FGFR inhibitors in FGFR1-amplified lung cancers with low FGFR1 protein expression. Oncogene 35, 3587-3597 (2016).

59. Singleton, K. R. et al. Kinome RNAi screens reveal synergistic targeting of MTOR and FGFR1 pathways for treatment of lung cancer and HNSCC. Cancer Res. 75, 4398-4406 (2015).

60. Lu, T. et al. The Hippo/YAP1 pathway interacts with FGFR1 signaling to maintain stemness in lung cancer. Cancer Lett. 423, 36-46 (2018).
61. Malchers, F. et al. Mechanisms of primary drug resistance in FGFR1-amplified lung cancer. Clin. Cancer Res. 23, 5527-5536 (2017).

62. Hayes, J., Peruzzi, P. P. \& Lawler, S. MicroRNAs in cancer: biomarkers, functions and therapy. Trends Mol. Med. 20, 460-469 (2014).

63. Gavine, P. R. et al. AZD4547: an orally bioavailable, potent, and selective inhibitor of the fibroblast growth factor receptor tyrosine kinase family. Cancer Res. 72, 2045-2056 (2012).

64. Yeh, T. C. et al. Biological characterization of ARRY-142886 (AZD6244), a potent, highly selective mitogen-activated protein kinase kinase 1/2 inhibitor. Clin. Cancer Res. 13, 1576-1583 (2007). 